\title{
Neurosífilis meningovascular y gomatosa cerebral concomitante, en paciente seronegativo para virus de inmunodeficiencia humana
}

\section{Meningovascular neurosyphilis and concomitant syphilitic gummata in hiv-seronegative patients}

Rodrigo Sánchez V. ${ }^{1}$, David Sáez M. ${ }^{1,2}$ y Tatiana Figueroa R. ${ }^{1-2}$

Neurosyphilis (NS) is caused by the presence of Treponema Pallidum (TP) spirochete within the Central Nervous System (CNS), mainly affecting the meninges and cerebrospinal fluid (CSF). $5 \%$ to $10 \%$ of untreated syphilitic patients are deemed to develop symptomatic NS ${ }^{1}$. Its incidence and clinical spectrum have changed over the years with prevalence of early clinical stages of meningitic and meningovascular (MV) NS and exceptional occurrence of late clinical stages (tabes dorsalis, general paresis, and gummata) in the age of antibiotics. The case under analysis deals with aggressive MV and concomitant brain gumma (BG) NS. The case subject is a human inmunodeficiency virus (HIV)-seronegative, 44-year-old woman with 2-year symptomatic latency. Her medical record showed recurrent sensorimotor vascular involvement, fast cognitive damage and chronic, daily cephalea. She met clinical diagnosis, cerebrospinal fluid and serologic criteria for NS. Brain computerized tomography (CT) and magnetic resonance $(M R)$ reported lenticulostriate artery infarction and bilateral ganglionic syphilitic gummata. She underwent Penicillin G-based treatment, making progress with neurological, cognitive-motor sequelae. Although NS has anticipated and speeded up its several clinical stages in connection with HIV/Syphilis co-infection, the peculiarity of this case is the concurrence of early and late NS manifestations in HIV-seronegative patient. The conclusion is that NS is a disease that still prevails and that appropriate diagnosis and treatment prevent irreversible neurological sequelae.

Key words: Neurosyphilis, treponema pallidum, meningovascular, cerebral gumma. Rev Chil Neuro-Psiquiat 2011; 49 (2): 165-170

\section{Introducción}

$\mathrm{L}$ a Sífilis es una enfermedad infecciosa sistémica crónica causada por la espiroqueta TP, frecuentemente trasmitida por contacto sexual. En la actualidad, su incidencia ha aumentado en paralelo a la pandemia del VIH/SIDA. En Estados Unidos, se estima una prevalencia de VIH en pacientes con Sífilis de $15,7 \%$, mientras que la prevalencia de NS en pacientes $\mathrm{VIH}$ es de $23,5 \%$, lo que muestra un sinergismo entre ambas patologías ${ }^{2,3}$.

Se denomina NS a la afectación del SNC por

Recibido: 14/12/2010

Aprobado: 11/03/2011

Departamento de Neurología Sur Hospital Barros Luco Trudeau.

2 Servicio de Neurología Hospital Barros Luco Trudeau. 
TP, incluyendo tanto las manifestaciones clínicas que de ella se derivan, en este caso NS sintomática, como aquellos casos asintomático ${ }^{1}$. La invasión del SNC ocurre en estadios tempranos de la infección, encontrándose TP en 25\% de los pacientes con sífilis temprana, independiente de su estado VIH, como también anomalías inflamatorias en LCR entre 40 a $70 \%$ de los pacientes con sífilis temprana asintomáticos ${ }^{4,5}$.

Las complicaciones neurológicas están asociadas a inflamación meningovascular y degeneración neuronal, comprometiendo cerebro, médula espinal y/o nervios periféricos. Las manifestaciones neurológicas de NS son heterogéneas y se observa una relación entre el tiempo transcurrido desde la infección primaria y el síndrome clínico desarrollado. Es así como la NS sintomática temprana, que incluye la forma meningítica aguda y MV, se presenta semanas hasta años después de la primo infección. En contraste, la NS tardía afecta primariamente parénquima de SNC y ocurre años a décadas después de la infección, incluyendo parálisis general, demencia rápidamente progresiva, tabes dorsal, atrofia del nervio óptico y gomas cerebrales (GC).

El diagnóstico de NS se basa en las características clínicas, serología y anomalías inflamatorias del LCR. El diagnóstico definitivo se realiza con una prueba treponémica en plasma reactiva, VDRL reactivo en LCR y la identificación microscópica de TP en tejidos con tinción de plata o anticuerpos fluorescentes. La especificidad de VDRL en LCR es $99 \%$ y su sensibilidad de $70 \%$, en etapa terciaria ${ }^{6,7}$. Las neuro-imágenes pueden tener valor diagnóstico en las formas MV y GC y en excluir otras patologías.

\section{Caso clínico}

Mujer de 44 años, tabáquica, sin otro factor de riesgo cerebro-vascular clásico. Con antecedente de contacto sexual sin protección, 5 años previo al inicio de los síntomas, con sujeto tratado por Lués. Su historia se inicia 2 años después, con disfunción neurológica focal sensitivo-motora recurrente, de perfil de instalación vascular, interpretada como infartos arteriales lacunares lentículo-estriados.
Evoluciona con rápido deterioro cognitivo y cefalea crónica diaria. Consultó un año después, por cuadro confusional agitado. Al ingreso destacaba, desorientada, pares craneales normales, hipertonía espástica de cuatro extremidades, hemiparesia proporcionada braquial y crural izquierda leve, hiperreflexia global, reflejo plantar extensor bilateral, hemihipoestesia termo-algésica izquierda y signos meníngeos altos. La TC de cerebro mostró lesiones focales hipodensas subcorticales (Figura 1a). El LCR era claro, 70 células de predominio monononuclear, proteínas $1,34 \mathrm{~g} / \mathrm{dl}$ y glucosa normal. Cultivo y tinta china negativo, ADA 10 U/L. El VDR en plasma fue reactivo $1 / 32$ y $1 / 8$ en LCR. La serología para VIH, HTLV-1, anticuerpos antinucleares, anti-DNA, anti-cardiolipinas, antiantígenos nucleares extractables y factor reumatoide resultó negativa. Hemograma y complemento, Ecocardiograma Transtorácico y Ecografía Doppler carotídeo fueron normales. La RM cerebral mostró lesiones de características quísticas granulomatosas a nivel de ganglios basales (Figural b, c, d, e, f). Recibió tratamiento con penicilina $G$ sódica endovenosa, en dosis de 24 millones de unidades diarias durante 21 días, continuado por penicilina benzatina 2,4 millones de unidades intramuscular semanal por tres semanas. La paciente evolucionó con regresión del cuadro meníngeo y con secuela cognitivo-motora, con fallas de memoria y fluencia verbal. Se realizó un control de LCR y RM tres meses después de tratamiento, el LCR fue de composición normal y VDRL no reactivo. La RM mostró leve disminución del tamaño de lesiones quísticas (Figura 2).

\section{Discusión}

Lo llamativo en este caso de NS, es la presentación concomitante de formas clínicas precoces y tardías. De curso agresivo, con compromiso MV y parenquimatoso en una paciente VIH (-). Situación común en pacientes VIH pero muy infrecuente en VIH (-). Es un hecho conocido, que la infección VIH cambia el perfil clínico de la NS, anticipando sus formas evolutivas y de curso más agresivo ${ }^{8-10}$.

La NS temprana afecta meninges y LCR, lo 


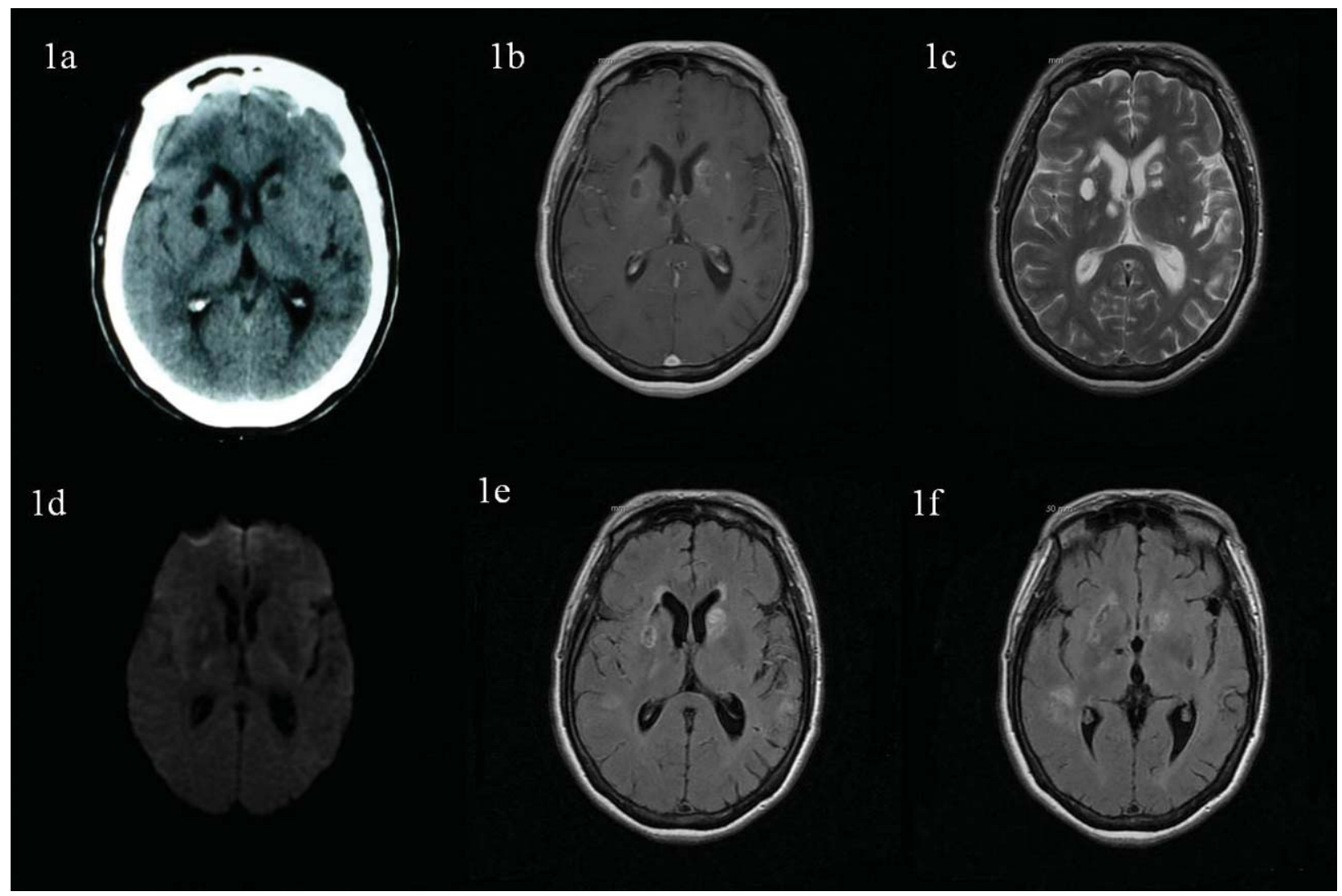

Figura 1. (a) TC axial, lesiones hipodensas en núcleos grises centrales, tálamo-capsular anterior y temporales izquierdas. (b) RM axial T1, lesiones redondeadas hipointensas con realce anular de contraste. (c) lesiones hiperintesas en T2 a nivel de núcleos lenticulares, ambas capsulas internas y tálamo derecho. (d) RM DWi, sin restricción a la difusión. (e) y (f) RM secuencia FLAIR, hiperseñal cortico-subcorticales en ambos lóbulos temporales.

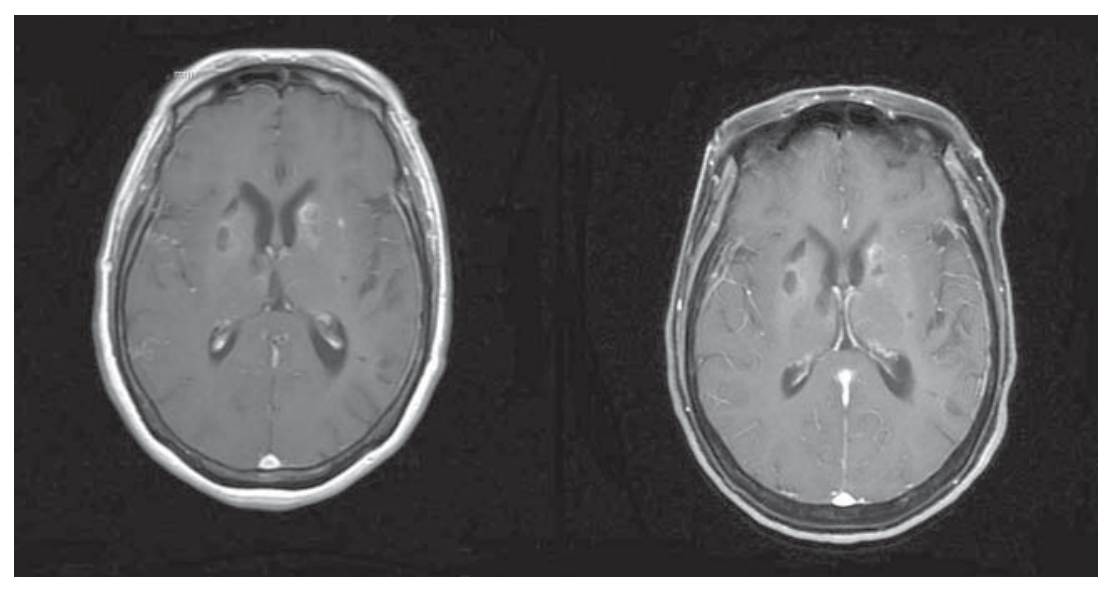

Figura 2. (a) RM T1 pre-tratamiento. (b) RM T1 post-tratamiento, muestra leve disminución del tamaño de lesiones quísticas que comprometían la cabeza del núcleo caudado y resolución de hiperintensidades temporales. 
que ocurre semanas a años después de la primo infección, manifestándose como meningitis o infartos cerebrales o espinales. En contraste, la NS tardía afecta el parénquima del SNC (tabes dorsal, demencia progresiva y gomas) y ocurre años a décadas después la infección primaria. Nuestro caso cumple criterios clínicos, licuolares y serológicos de NS. Las manifestaciones neurológicas principales fueron focalidad sensitivo-motor de perfil vascular, deterioro cognitivo-motor, cefalea y episodios de confusión, con una latencia sintomática de 3 años. Si bien, esto se puede ver en la NS MV, las neuroimágenes mostraron lesiones quísticas granulomatosas de localización atípica (ganglios basales), compatibles con gomas sifilíticas. La forma MV es la presentación más frecuente en individuos inmunocompetentes, con una latencia sintomática de meses hasta 7 años ${ }^{11}$. Puede producir infartos en arterias de pequeño, mediano y gran tamaño, afectando con mayor frecuencia la arteria cerebral media, si bien pueden ser otros territorios vasculares cerebrales y medulares. Histopatológicamente se caracteriza por un infiltrado celular inflamatorio linfoplasmocitario de leptomeninges, arterias y parénquima superficial, produciendo una endoarteritis obliterativa, caracterizada por proliferación fibroblástica subintimal y daño arterial irreversible de fibras musculares y elásticas, llevando a una estenosis vascular causando infartos cerebrales ${ }^{5,12,13}$. El cuadro clínico incluye elementos meníngeos y focalidad neurológica con un perfil de instalación que no difiere en su naturaleza de otras lesiones vasculares cerebrales. El diagnóstico diferencial principal es la meningitis TBC y procesos vasculíticos.

Las GC han sido clásicamente descritas como marcadoras de Sífilis terciaria, con una latencia sintomática de alrededor 15 años. En la era antibiótica, su ocurrencia es excepcional, su frecuencia fluctúa entre 0,1 a $0,5 \%$ en series de tumores intracraneales $^{14}$. Histopatológicamente corresponden a una lesión inflamatoria granulomatosa, caracterizada por infiltrado celular linfoplasmocitario rodeando a un núcleo necrótico con macrófagos y fibroblastos. Las GC tienen una localización preferente en las convexidades cerebrales. Otras locali- zaciones comunicadas incluyen glándula pituitaria, ganglios basales, tronco, cerebelo, tálamo, hipotálamo y ángulo ponto-cerebeloso. Su presentación clínica habitual es de perfil tumoral y convulsiones.

En pacientes con NS, los hallazgos en TC y RM cerebral son inespecíficos. Se han reportado: atrofia cortico-subcortical, infartos corticales y subcorticales, lesiones inespecíficas de sustancia blanca, hipertintensidades mesotemporales, reforzamiento lepto meníngeo, arteritis y gomas ${ }^{15-17}$. Las GC se presentan como lesión nodular única o múltiple, su tamaño varía de milímetros hasta $10 \mathrm{~cm}^{18}$. En TAC aparecen lesiones focales hipodensas que pueden acompañarse de reforzamiento de contraste y/o efecto de masa. En RM, en secuencias T1 y T2 muestra imágenes hipointensas e hiperintensas respectivamente y reforzamiento anular de contras$\mathrm{te}^{18}$. Nuestro caso, clínicamente presentó un perfil temporal vascular a diferencia del perfil tumoral habitual de las gomas de localización cortical. La RM evidenció lesiones quístico-granulomatosas ganglionares, cuya localización es atípica y de características similares a lo reportado en la literatura. Con el tratamiento hubo regresión de cuadro meníngeo, sin embargo, la paciente quedo con daño neurológico cognitivo y motor irreversible, lo que estimamos fue por lo tardío del diagnóstico. La terapia esta dirigida a detener la progresión del daño vascular y neuronal, pero tiene escaso efecto en el daño ya establecido. En la actualidad la sífilis es una enfermedad re-emergente, sin embargo, hemos dejado de pesar en la sífilis como causa de infartos cerebrales en paciente jóvenes, situación que debiésemos remediar.

\section{Conclusión}

El caso analizado corresponde a una NS sintomática de curso anticipado y agresivo en sus formas clínicas MV y GC, en la actualidad infrecuente. Lo destacable del caso, es que si bien la NS actualmente ha anticipado sus diferentes formas evolutivas debido a la co-infección Sífilis/VIH, constituye una excepción en pacientes VIH (-), que es la situación del caso en comento. 


\begin{abstract}
Resumen
La Neurosifilis (NS) es causada por la invasión del Sistema Nervioso Central (SNC) por la espiroqueta Treponema Pallidum (TP), afectando primariamente las meninges y líquido cefalorraquídeo. Entre 5 a 10\% de los pacientes sifiliticos no tratados desarrollarán una NS sintomática. ${ }^{1}$ Su incidencia y espectro clínico ha cambiado a lo largo del tiempo, siendo las formas clínicas precoces meníngea y meningovascular (MV) las más prevalentes. En contraste, las formas tardías (tabes dorsal, parálisis general y gomas) son de ocurrencia excepcional en la era antibiótica. Se analiza un caso de NS menigovascular y gomas cerebrales concomitantes, de curso clínico agresivo. En una mujer de 44 años, seronegativa para virus de inmunodeficiencia humana (VIH), con latencia sintomática de dos años. Presentaba una historia de focalidad sensitivo-motor de perfil vascular recurrente, rápido deterioro cognitivo-motor y cefalea crónica diaria. Cumplía criterios diagnósticos clínicos, licuorales y serológicos para NS. La Tomografía computada (TC) y Resonancia Magnética (RM) cerebral mostró infartos arteriolares lentículoestriados y gomas sifiliticas ganglionares bilaterales. Recibió tratamiento con Penicilina G, evolucionando con secuelas neurológicas cognitivas-motoras. Si bien, la NS actualmente, ha anticipado y acelerado sus diferentes formas clinicas en relación a co-infección VIH/Sífilis. Lo llamativo de este caso, es la presentación concomitante de con manifestaciones precoces y tardías de NS en paciente VIH seronegativo. Se concluye que la NS sigue siendo una enfermedad vigente y su diagnóstico y tratamiento oportuno previene secuelas neurológicas irreversibles.
\end{abstract}

Palabras clave: Neurosifilis, treponema pallidum, meningovascular, goma sifilitica.

\section{Referencias}

1. Conde-Sendín MA, Hernández-Fleta JL, CárdenesSantana MA, Amela-Peris R. Neurosífilis: formas de presentación y manejo clínico. Rev Neurol 2002; 35: 380-6.

2. Blocker ME, Levine WC, St Louis ME. HIV prevalence in patients with syphilis, United States. Sex Transm Dis 2000; 27: 53-9.

3. Bordon J, Martínez-Vázquez C, Álvarez M, et al. Neurosyphilis in HIV-infected patients. Eur J Clin Microbiol Infect Dis 1995; 14: 864-9.

4. Marra CM. Neurosyphilis. Curr Neurol Neurosci Rep 2004; 4: 435-40.

5. Núñez DC, Verdugo LR, Cartier RL. Meningovasculitis luética de la médula espinal: "Spinal vascular syphilis of Singer". Rev Chil Neuro-Psiquiatr 2009; 47: 138-43.

6. Lynn WA, Lightman, S. Syphilis and HIV: a dan- gerous combination. Lancet Infect Dis 2004; 4: 456-66.

7. Larsen SA, Steiner BM, Rudolph AH. Laboratory diagnosis and interpretation of tests for syphilis. Clin Microbiol Rev 1995; 8: 1-21.

8. Lasso BM, Balcells ME, Fernández SA, Gaete GP, Serri VM, Pérez GJ, et al. Neurosífilis en pacientes portadores y no portadores de VIH: Descripción y comparación de dos cohortes históricas. Rev Chil Infectol 2009; 26: 540-7.

9. Funnye AS, Akhtar AJ. Syphilis and human immunodeficiency virus co-infection. J Natl Med Assoc 2003; 95: 363-82.

10. Johns DR, Tierney M, Felsenstein D. Alteration in the natural history of neurosyphilis by concurrent infection with the human immunodeficiency virus. N Engl J Med 1987; 316: 1569-72.

11. Conde-Sendín MA, Amela-Peris R, Aladro-Benito Y, Maroto AA. Current clinical spectrum of neu- 
rosyphilis in immunocompetent patients. Eur Neurol 2004; 52: 29-35.

12. Hook EW, Marra CM. Acquired syphilis in adults. N Engl J Med 1992 16; 326: 1060-9.

13. Carr J. Neurosyphilis. Practical Neurology. 2003; 3:328-31.

14. Fargen KM, Alvernia JE, Lin CS, Melgar M. Cerebral syphilitic gummata: a case presentation and analysis of 156 reported cases. Neurosurgery 2009; 64: 568-76.

15. Peng F, Hu X, Zhong X, Wei Q, Jiang Y, Bao J, et al. $\mathrm{CT}$ and MR findings in HIV-negative neurosyphi- lis. Eur J Radiol 2008; 66: 1-6.

16. Brightbill TC, Ihmeidan IH, Post MJ, Berger JR, Katz DA. Neurosyphilis in HIV-Positive and HIVNegative Patients: Neuroimaging Findings AJNR Am J Neuroradiol 16: 703-11.

17. Gürses C, Bilgiç B, Topçular B, Tuncer OG, AkmanDemir G, Hanağasi $\mathrm{H}$, et al. Clinical and magnetic resonance imaging findings of HIV-Negative patients with neurosyphilis. J Neurol 2007; 254: 368-74.

18. Agrons GA, Han SS, Husson MA, et al. MR imaging of cerebral gumma. AJNR Am J Neuroradiol 1991; 12: 80-1.

Correspondencia:

Dr. David Sáez Méndez

Servicio de Neurología Hospital Barros

Luco Trudeau, Gran Avenida 3204, San Miguel.

Teléfono: 3948941

E-mail: davidsaez@vtr.net 